\title{
LETTER
}

\section{Comparison of ultrasound-guided modified Seldinger technique versus blind puncture for peripherally inserted central catheter: a meta-analysis of randomized controlled trials}

\author{
ZhanZhan Li and LiZhang Chen*
}

The peripherally inserted central catheter (PICC) has been used for central venous pressure monitoring and building effective infusion routes in a critical care setting. The traditional blind puncture technique for PICC has been challenging. The ultrasound-guided modified Seldinger technique (MST) has been used as an adjunct for central venous catheter and has shown significant benefits over the traditional blind puncture. However, despite clear advantages, complications of ultrasoundguided MST for PICC still occur. We conducted a metaanalysis of randomized controlled trials (RCTs) to compare the ultrasound-guided MST and traditional blind puncture techniques for PICC.

PubMed and CNKI (Chinese database) were searched for RCTs comparing the ultrasound-guided MST and traditional palpation for radial artery catheterization. Differences were expressed as relative risks (RRs) with 95\% confidence intervals (CIs) for dichotomous outcomes. The fixed-effects model or random-effects model was used, depending on whether heterogeneity existed among studies. Heterogeneity among studies was examined with Cochran's $Q$ statistic $(P<0.1)$ and the $I^{2}$ statistic. A two-tailed $P$ value of less than 0.05 was considered a significant level except for where a certain $P$ value has been given. Six RCTs enrolling 726 patients (367 trials and 359 controls) were included in the meta-analysis [1-6]. Three studies $[1,3,4]$ report that the first-attempt success rate of ultrasound-guided MST was superior to that of the traditional blind puncture, and three studies $[2,5,6]$ report that the first-attempt success rate was similar for the two techniques. The meta-analysis indicated

\footnotetext{
* Correspondence: liche4005@126.com

Department of Epidemiology and Health Statistics, School of Public Health, Central South University, Xiangya Road, Kaifu District, Changsha, Hunan
} Province, 410078, China

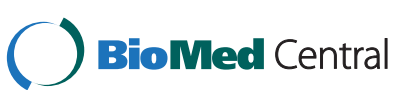

(c) $2015 \mathrm{Li}$ and Chen; licensee BioMed Central. This is an Open Access article distributed under the terms of the Creative Commons Attribution License (http://creativecommons.org/licenses/by/4.0), which permits unrestricted use, distribution, and reproduction in any medium, provided the original work is properly credited. The Creative Commons Public Domain Dedication waiver (http://creativecommons.org/publicdomain/zero/1.0/) applies to the data made available in this article unless otherwise stated. that ultrasound-guided MST was not significantly associated with improvement in first-attempt success $(\mathrm{RR}=1.07$, 95\% CI 0.99 to $1.16, P=0.090$ ) compared with the traditional blind puncture, but ultrasound-guided MST significantly reduced the incidence of complication after surgery ( $R R=0.24,95 \% \mathrm{CI} 0.08$ to $0.76, P=0.015$ ).

In summary, ultrasound-guided MST is superior to the traditional blind puncture technique for PICC, especially for postoperative complications. It should be generalized to clinical application for PICC.

\section{Abbreviations}

Cl: Confidence interval; MST: Modified Seldinger technique; PICC: Peripherally inserted central catheter; RCT: Randomized controlled trial; RR: Relative risk.

\section{Competing interests}

The authors declare that they have no competing interests.

\section{Authors' contributions}

ZZL and LZC conceived the study, participated in the design, collected the data, and drafted the manuscript. Both authors read and approved the final manuscript.

\section{Acknowledgments}

This work was supported by a Hunan Province Science and Technology Project (2014SK2013) and fundamental research funds for the central

Published online: 14 February 2015

\section{References}

1. Knebel P, Fischer L, Huesing J, Hennes R, Buchler MW, Seiler CM. Randomized clinical trial of a modified Seldinger technique for open central venous cannulation for implantable access devices. Br J Surg. 2009; 96:159-65.

2. Wan $\mathrm{YH}$, Tan F, Chen Q, Wang Q. Effects of modified Seldinger technique in vascular ultrasound-guided PICC placement of upper arm. Nurs J Chin PLA. 2010; 27:1507-8.

3. Bao $A Q$, Wen $Q$, Liu WH. Using ultrasound-guided modified Seldinger technique for PICC insertion. J Nurs Sci. 2010;25:57-8.

4. Ding RM, Yang Y, Wang P, Ma L. Ultrasound-guided modified Seldinger technique PICC and conventional PICC in the study of difficult comparison universities of Central South University (2014zzts069). 
5. Li J, Fan YY, Xin MZ, Yan J, Hu W, Huang WH. A randomised, controlled trial comparing the long-term effects of peripherally inserted central catheter placement in chemotherapy patients using B-mode ultrasound with modified Seldinger technique versus blind puncture. Eur J Oncol Nurs. 2014;18:94-103.

6. Liu ZM, Liu CY, Shi SQ, Zhang J. Clinical study of the comparison of ultrasound-guided modified Seldinger technique and blind wear of placement of PICC. J Nurs Train. 2012; 27:773-5. 